Reprod. Nutr. Dévelop., 1987, 27 (3), 733-743.

\title{
Effet de l'addition d'un concentré riche en fibres dans une ration à base de foin, distribuée à deux niveaux alimentaires chez la lapine adulte. 1 . Temps de séjour moyen des aliments
}

\author{
T. GIDENNE, C. PONCET $(*)$, L. GOMEZ $(*)$
}

Laboratoire de Recherches sur l'Elevage du Lapin, I.N.R.A., B.P. 27, 31326 Castanet-Tolosan Cedex, France

(*) Laboratoire de la Digestion, I.N.R.A.,

Theix, 63122 Ceyrat

Summary. Addition of a fibre-rich concentrate to a hay-based diet offered at two levels of feeding to adult rabbits. 1. Mean retention time of digesta.

The influence of adding a fibre rich concentrate feed (wheat bran or beet pulp) to a hay-based diet (orchard grass-lucerne mixture ; 50/50) upon the rate of passage of digesta of these two fractions (hay and concentrate) was measured in three groups of 8 adult doe rabbits (White New Zealand $\times$ California). The three diets composed of either $90 \%$ hay, or hay + bran or hay + pulp were offered at two levels of feeding, i.e. ad libitum and $2 / 3$ ad libitum. The retention time of the two main fractions was measured by means of two tracers, i.e. ytterbium-169 bound to hay and cerium-141 to bran or pulp.

Animals fed on the $90 \%$ hay diet excreted more than $90 \%$ of the tracer during the first $24 \mathrm{~h}$. The mean retention time (MRT) of this diet was the shortest (on an average $14.8 \mathrm{~h})$. The addition of pulp or bran increased the MRT of the diet by 2 and $4 \mathrm{~h}$, respectively. Feed restriction led to a 5 to $7 \mathrm{~h}$ earlier period of caecotrophy with a subsequent lengthening of the MRT by 2 to $4 \mathrm{~h}$ according to groups.

The two hay + " concentrate " diets gave almost the same ${ }^{169} \mathrm{Yb}$ and ${ }^{141} \mathrm{Ce}$ excretion curves. Thus, the mean retention time seems to be the same for digesta from hay and " concentrate " (non significant deviations).

\section{Introduction.}

Plusieurs auteurs ont étudié le transit digestif chez le Lapin en suivant l'excrétion fécale d'un marqueur.

Outre une meilleure connaissance de la physiologie digestive du lapin, ces mesures de transit apportent au plan zootechnique des pécisions sur les relations entre le temps de séjour des aliments et leur valeur alimentaire, le principe suivant étant généralement admis : un temps de séjour bref est lié à une digestibilité plus faible de la ration.

Ainsi, Laplace et Lebas (1975) mesurent la vitesse de transit de la ration dans son ensemble, à l'aide d'une dose de cérium déposée sur une fraction de l'aliment ou administrée sous forme d'une solution. Uden et al. (1982) étudient chez le lapin le transit d'un foin de fléole additionné d'un concentré, à l'aide du chrome 
mordancé sur le foin. Mais nous n'avons pas d'informations sur un éventuel transit différentiel des principales matières premières d'un aliment composé. Notre étude se propose donc de mesurer simultanément le temps de séjour moyen dans l'ensemble du tube digestif pour les deux principales matières premières d'une ration : un foin et un aliment " concentré " riche en fibres (pulpe de betterave ou non de blé).

Ledin (1984) remarque, chez des lapins nourris avec un aliment composé, que le rationnement entraîne un allongement du temps de transit. C'est pourquoi, dans notre étude, l'effet éventuel de la nature du " concentré " sur la vitesse de passage du foin et sur celle du "concentré " lui même, est mesuré à deux niveaux d'alimentation.

Le transit des deux principales matières premières foin et "concentré " est suivi à l'aide de deux marqueurs de la famille des terres rares : I'ytterbium fixé sur le foin et le cérium fixé sur le " concentré ". Les terres rares, déjà employées chez le Lapin par Laplace et al. (1974) sont actuellement les plus utilisés, parmi les marqueurs de la phase particulaire. Dans les meilleures conditions d'emploi, ces marqueurs sont préalablement fixés sélectivement sur les sites à forte affinité de l'aliment débarrassé de ses constituants non pariétaux (Ellis et Beever, 1985). Cette technique n'a pas encore fait l'objet d'étude chez le Lapin, mais elle a déjà été utilisée avec les mêmes aliments chez le Ruminant (Poncet et al., 1987a).

\section{Matériel et méthodes.}

1) Schéma expérimental et type d'aliment. - Trois rations ont expérimentées ; leur composition est définie au tableau 1.

La ration "foin " est composée de $90 \%$ d'un foin de luzerne et dactyle (50/50). Dans les deux autres rations, le foin a été mélangé dans un rapport de $2 / 3$ à un aliment " concentré " riche en parois : la pulpe de betterave (lot FoinPulpe), ou le son de blé (lot Foin-Son). Le foin et le "concentré " ont été broyés, mélangés aux autres éléments de la ration, puis agglomérés sous forme de granu-

TABLEAU 1

Composition des trois aliments expérimentaux (en \% MS).

\begin{tabular}{|c|c|c|c|}
\hline \multirow[b]{2}{*}{ Composants } & \multicolumn{3}{|c|}{ Ration } \\
\hline & $\begin{array}{c}1 \\
\text { « Foin } »\end{array}$ & «Foin-Pulpe » & «Foin-Son " \\
\hline Foin de luzerne et dactyle $(50 / 50)$. & 90,0 & 36,0 & 36,0 \\
\hline Son de blé tendre $\ldots \ldots \ldots \ldots \ldots \ldots$ & - & - & 54,0 \\
\hline Pulpe de betterave $\ldots \ldots \ldots \ldots \ldots \ldots$ & - & 54,0 & - \\
\hline Farine de poisson « 72 maigre $" \ldots \ldots \ldots$ & 4,0 & 4,0 & 4,0 \\
\hline Amidon de maïs $\ldots \ldots \ldots \ldots \ldots \ldots \ldots$ & 4,5 & 4,5 & 4,5 \\
\hline $\mathrm{NaCl} \ldots \ldots \ldots \ldots \ldots \ldots \ldots$ & 0,5 & 0,5 & 0,5 \\
\hline Composé vitaminique et minéral (1) .... & 1,0 & 1,0 & 1,0 \\
\hline
\end{tabular}

(1) Colin, Arkhurst et Lebas, 1973. 
lés. Au cours d'une première période (P1), les rations ont été distribuées ad libitum. Pendant une seconde période (P2), le niveau d'ingestion a été limité individuellement aux $2 / 3$ de la consommation ad libitum, mesurée pendant $\mathrm{P} 1$, excepté dans le cas de la ration foin-son (à $80 \%$ de l'ingéré à volonté). Les rations ont été distribuées à 3 lots de 8 lapines adultes, métis (Néozélandais $\times$ Californiens), maintenues en cage à digestibilité.

2) Technique de marquage des aliments et mode d'administration. - L'expérience décrite ici appartient à un projet plus large, dont l'objectif était de comparer la digestibilité et le temps de séjour moyen d'aliments riches en fibres, chez différentes espèces d'herbivores. Dans le cas d'animaux qui n'étaient pas en production, tel que les lapins adultes, des marqueurs radioactifs ont été employés : ${ }^{169} \mathrm{Yb}$ pour le foin ; ${ }^{141} \mathrm{Ce}$ pour le « concentré ".

Le marquage des aliments a été effectué selon le principe proposé par Ellis et Beever (1985). L'objectif est de fixer les marqueurs sur les sites de fixation à forte affinité. Les principales étapes du marquage sont les suivantes:

- lavage de chaque matière première, afin d'en ôter les constituants solubles ;

- trempage dans une solution contenant le radio-élément (12 h pour le foin, $24 \mathrm{~h}$ pour la pulpe ou le son);

- trempage durant $2 \mathrm{~h}$ dans une solution contenant un chelatant lacide citriquel, qui décroche les particules de marqueur faiblement liées à la matière première ;

- lavage et séchage de l'aliment, puis préparation des doses à administrer.

Une dose de marqueur correspond pour chaque animal à environ 37 à 74 kBq de ${ }^{169} \mathrm{Yb}$ ou de ${ }^{141} \mathrm{Ce}$ contenues dans environ $5 \mathrm{~g}$ d'aliment traité.

Les particules d'aliment marqué, correspondant à une dose, ont été broyées et agglomérées sous forme d'un granulé. Celui-ci a été déposé dans la gueule du lapin qui est surveillé jusqu'à déglutition.

3) Mesures de transit. - Les mesures de transit digestif ont été effectuées après une phase d'accoutumance au régime de deux semaines pour $P 1$ et $d^{\prime} u n e$ semaine pour $P 2$. La dose d'aliment marqué a été administrée en une seule fois, le matin entre 8 h 30 et 9 h 30 (to).

L'excrétion des marqueurs a été suivie par récoltes (manuelles) totales des fèces. Les récoltes ont débuté à $11 \mathrm{~h} 30$ puis elles ont été effectuées selon le protocole suivant : toutes les $2 \mathrm{~h}$ jusqu'à la $1^{\mathrm{o}}$ heure; toutes les $4 \mathrm{~h}$ jusqu'à la $38^{e}$ heure ; toutes les $8 \mathrm{~h}$ jusqu'à la $54^{\mathrm{e}}$ heure.

Après chaque récolte, les fèces ont été pesées et séchées pendant $48 \mathrm{~h}$ à $80^{\circ} \mathrm{C}$ (détermination de la matière sèche). Puis elles ont été soumises au comptage de la radioactivité.

4) Exploitation des résultats. - Le temps de séjour moyen dans l'ensemble du tube digestif (TSM) a été calculé selon la méthode des moments (Blaxter et al., 1956) :

$$
\operatorname{TSM}=\frac{\Sigma \mathrm{Ci} \mathrm{Qi} \mathrm{ti}}{\Sigma \mathrm{Ci} \mathrm{Qi}}
$$


$\mathrm{Ci}=$ concentration du marqueur dans les fèces ; $\mathrm{Q} i=$ quantité sèche de fèces excrétées ; $\mathrm{ti}=$ temps moyen écoulé depuis l'administration.

La méthode des moments déjà employée chez le mouton ou le porc (Thielemans et al., 1978), tient compte des quantités de marqueur excrétées et du moment de l'excrétion. Les TSM sont calculés à partir de récoltes réparties sur $54 \mathrm{~h}$, soit plus de deux cycles digestifs. Cela permet d'intégrer les quantités de marqueur éventuellement recyclées par la caecotrophie, dont l'excrétion dans les crottes dures est plus tardive. II s'agit donc, pour le lapin, des temps de séjour moyen pour l'ensemble du tube digestif, en incluant la pratique de la caecotrophie.

L'analyse statistique des résultats a été effectuée par analyse de variance à un ou plusieurs facteurs de variation.

\section{Résultats.}

L'administration de la dose de marqueur par ingestion forcée d'un "granulé " a entraîné un stress plus ou moins important chez l'animal. Aussi, les résultats ne concernent que les animaux ayant eu une consommation d'aliment et une excrétion fécale, en période de mesure de transit, identiques à celles mesurées en période d'accoutumance. Nous éviterons ainsi tout artefact dû aux conditions d'ingestion du marqueur.

Le poids vif moyen des animaux n'a pas significativement différé entre les lots, que ce soit en période 1 ou 2 (tabl. 2). Par contre, l'ingestion à volonté (P1) a varié significativement $(P<0,01)$ selon le lot : ainsi la consommation pour la ration 1 (90\% de Foin) a atteint environ $170 \mathrm{~g}$ de $M S / \mathrm{j}$, mais n'a pas dépassé

TABLEAU 2

Caractéristiques zootechniques des animaux (moyenne \pm écart type).

\begin{tabular}{|c|c|c|c|c|c|c|c|c|}
\hline \multirow[b]{2}{*}{ Lots } & \multicolumn{4}{|c|}{ Alimentation à volonté } & \multicolumn{4}{|c|}{ Alimentation rationnée } \\
\hline & $n$ & $\begin{array}{l}\text { Poids vif } \\
\text { initial (g) }\end{array}$ & $\begin{array}{l}\text { Ingéré } \\
\sec (g / j)\end{array}$ & $\begin{array}{c}\text { Excrété } \\
\text { sec fécal } \\
(\mathrm{g} / \mathrm{j})\end{array}$ & $n$ & $\begin{array}{c}\text { Ingéré } \\
\sec (g / j)\end{array}$ & $\begin{array}{c}\text { Excrété } \\
\text { sec fécal } \\
(\mathrm{g} / \mathrm{j})\end{array}$ & $\begin{array}{l}\text { Poids vif } \\
\text { final }(\mathrm{g})\end{array}$ \\
\hline Foin & 4 & 4139 & $168,9^{a}$ & $87,4^{a}$ & 4 & $110,2^{\mathrm{a}}$ & $71,1^{a}$ & 3959 \\
\hline rom & & $\pm \quad 479$ & $\pm \quad 15,6$ & $\pm 12,1$ & & $\pm 9,2$ & $\pm 7,3$ & $\pm \quad 429$ \\
\hline Foin + Pulpe & 3 & 3980 & $134,1^{\mathrm{ab}}$ & $46,3^{b}$ & 5 & $85,7^{b}$ & $38,4^{b}$ & 3924 \\
\hline rum t r uाpe & 3 & $\pm \quad 646$ & $\pm 18,2$ & $\pm \quad 4,5$ & 5 & 3,6 & $\pm 10,0$ & $\pm \quad 252$ \\
\hline Foin + Son & 3 & 4260 & $102,6^{a}$ & $52,6^{b}$ & 5 & $82,4^{b}$ & $53,3^{b}$ & 3939 \\
\hline roll t som & 0 & $\pm \quad 396$ & $\pm 25,2$ & $\pm \quad 8,2$ & 2 & 5,5 & $\pm 4,0$ & $\begin{array}{l} \pm 29 \\
\end{array}$ \\
\hline Test F & & NS & ** & ** & & ** & $* *$ & NS \\
\hline
\end{tabular}

N.B. : valeurs obtenues durant les mesures de transit (2 jours).

$a, b$ : les moyennes ayant une lettre en commun ne diffèrent pas au seul $P<0.05$ (Test de Newmann et Keuls).

$\mathrm{n}$ : effectifs contrôlés.

** : écart significatif au seuil $P<0,01$. 
$103 \mathrm{~g} / \mathrm{j}$ pour la ration foin + son. Pendant la seconde période, la consommation a été effectivement rationnée à $65 \%$ de la consommation à volonté pour les lots "Foin" et "Foin + Pulpe ". Le lot "Foin + Son " a présenté une ingestion volontaire assez faible (P1). Aussi, dans le but d'éviter une perte de poids trop importante au cours de $\mathrm{P} 2$, le rationnement appliqué a-t-il été moins sévère : $80 \%$ de l'ingestion mesurée pendant la période ad libitum.

Au cours de la journée, la répartition des excrétions fécales varie fortement selon l'individu considéré. Aussi avons nous présenté, à titre d'illustration, les courbes de concentrations ( $\mathrm{Ci}$ ) et d'excrétions cumulées (CumEx) des marqueurs, d'un individu représentatif de chaque lot (fig. 1). Dans le but de comparer les trois lots, l'excrétion cumulée du marqueur de chaque individu a été exprimée en pourcentage de son excrétion totale. De mêmé, la concentration en marqueur dans les fèces a été exprimée en pourcentage de la concentration maximale. L'axe des abscisses correspond au temps (en heures) écoulé depuis le moment de l'administration du marqueur (to).

En période d'alimentation à volonté, la concentration en $\mathrm{Yb}$ pour le lot "Foin" (fig. 1) a montré une croissance et une décroissance très rapide. En moyenne pour ce lot, la quantité de marqueur excrétée pendant les premières $24 \mathrm{~h}$ (E) a atteint $92 \%$. Dans le cas de chacun des deux lots « Foin + Concentré ", les courbes d'évolution des concentrations et d'excrétion cumulée des deux marqueurs ( $\mathrm{Yb}$ et $\mathrm{Ce}$ ) sont parallèles. Les valeurs de $\mathrm{E}$ (tabl. 3) n'ont pas différé significativement. Les animaux du lot " Foin + Son " ont excrété à peine $84 \%$ des marqueurs pendant le premier jour. Les animaux ayant reçu la ration "Foin + Pulpe 》 ont présenté une excrétion intermédiaire (fig. 1), puisque la valeur moyenne de $\mathrm{E}$ a été de $87,0 \%$. La pratique de la caecotrophie a lieu dans la matinée ; dans nos enregistrements, elle correspond à une période d'absence d'excrétion fécale. La courbe d'excrétion cumulée présente alors un plateau dans

TABLEAU 3

Quantité cumulée de marqueur excrétée (E) pendant les $24 \mathrm{~h}$ suivant l'administration du marqueur (Moyenne \pm écart type, exprimée en \% du total excrété).

\begin{tabular}{lccccccc}
\hline \multirow{2}{*}{ Lots } & \multicolumn{2}{c}{ Alimentation à volonté } & \multicolumn{2}{c}{ Alimentation rationnée } & \multicolumn{2}{c}{ Effet niveau alimentaire } \\
\cline { 2 - 7 } & $\mathrm{Yb}$ & $\mathrm{Ce}$ & $\mathrm{Yb}$ & $\mathrm{Ce}$ & $\mathrm{Yb}$ & $\mathrm{Ce}$ \\
\hline Foin & $91,7 \pm 3,9^{\mathrm{a}}$ & - & $74,6 \pm 7,1^{\mathrm{a}}$ & & $* *$ & - \\
\hline Foin + Pulpe & $87,0 \pm 5,2^{\mathrm{ab}}$ & $86,6 \pm 6,6$ & $56,2 \pm 11,4^{\mathrm{b}}$ & $61,6 \pm 15,2$ & $* *$ & $*$ \\
\hline Foin + Son & $83,7 \pm 5,5^{\mathrm{b}}$ & $82,3 \pm 6,6$ & $47,8 \pm 6,4^{\mathrm{b}}$ & $53,0 \pm 2,4$ & $* *$ & $*$ \\
\hline Effet ration & $*$ & NS & $* *$ & NS & & \\
\hline
\end{tabular}

(Résultats de l'analyse de variance à un facteur, " ration " ou " niveau alimentaire »).

$a, b$ : pour l'effet ration, les moyennes ayant une lettre en commun ne diffèrent pas au seuil $P<0,05$ (Test de Newmann et Keuis).

* : écart significatif au seuil $P<0,05$.

${ }^{*}$ : écart significatif au seuil $P<0,01$. 
la matinée du second jour (fig. 1 : lot "Foin + Son ", P1). Après cette phase, la courbe de concentration en $\mathrm{Yb}$ ou en Ce présente un rebond.
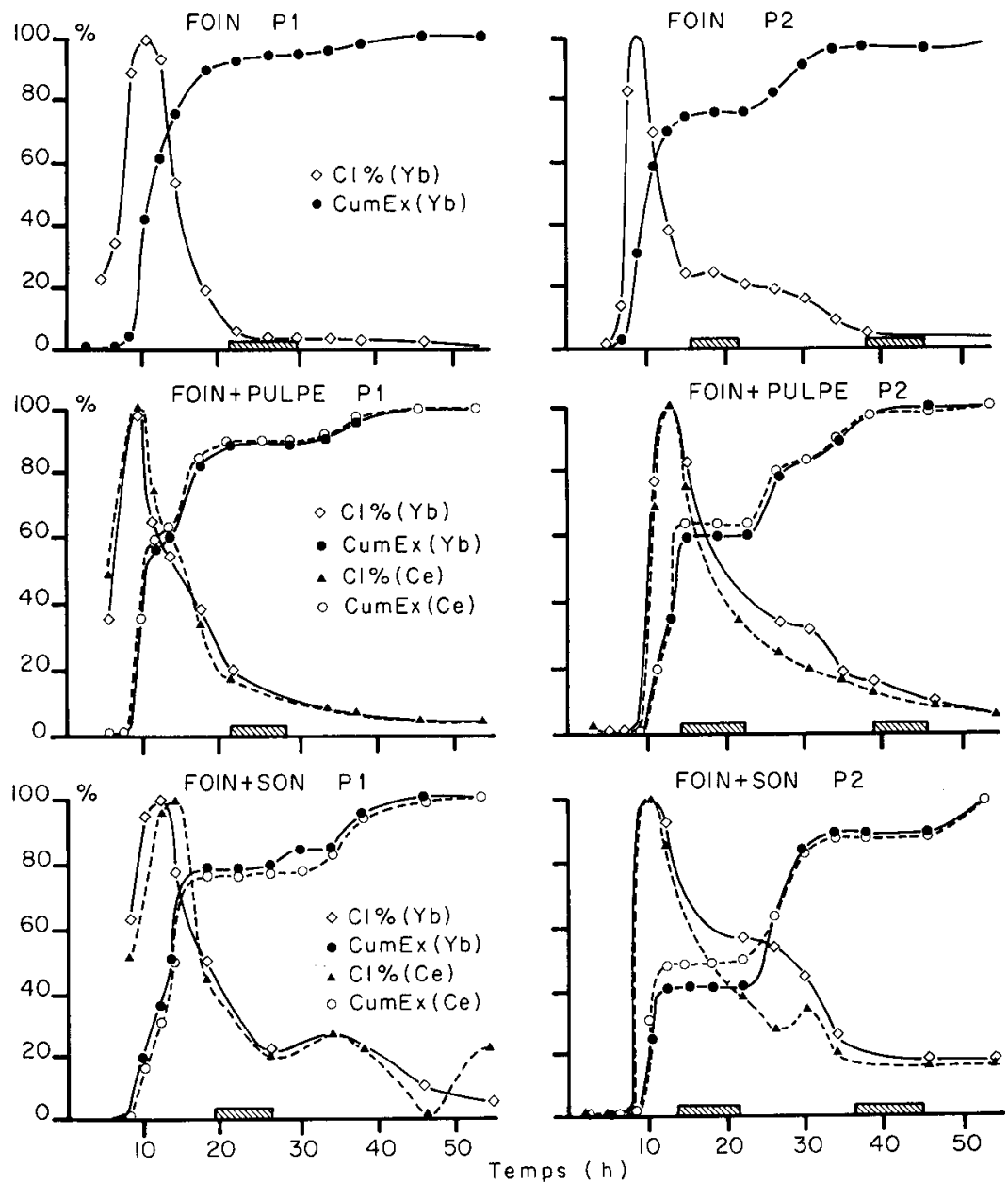

FIG. 1. - Evolution de la concentration fécale en marqueur ( $\mathrm{Ci}$ en $\%$ de $\mathrm{Ci}$ max.) et de son excrétion cumulee (CumEx) en fonction du temps (exemple de courbes individuelles d'excrétion).

vtterbium $(\mathrm{Yb})$ : cérium $(\mathrm{Ce})$ : caecotrophie :

NB : Ci est exprimé en \% de la concentration maximum, et CumEx est exprimé en \% du total excrété.

Quand les animaux ont été rationnés, la période de caecotrophie a été avancée (en moyenne) de $5 \mathrm{~h}$ à $7 \mathrm{~h}$. Ainsi, la quantité de marqueur excrétée en $24 \mathrm{~h}$ (E) n'est plus que de $75 \%$ (écart significatif par rapport à $\mathrm{P} 1$ ) dans le cas du lot "Foin ". Un plateau se dessine nettement sur la courbe d'excrétion cumulée (fig. 1), suivi d'un léger retard des concentrations en $\mathrm{Yb}$. Ce phénomène est plus marqué pour le lot " Foin + Son " $(E=48 \%$ en moyenne). Le lot " Foin + Pulpe » a une situation intermédiaire : $E=56 \%$ en moyenne. Chez les lapins recevant 
du son, un second plateau d'excrétion se dessine $35 \mathrm{~h}$ après administration du marqueur; il correspond à la période de caecotrophie du nycthémère suivant $\left(2^{e}\right.$ jour de collecte).

L'analyse des valeurs du temps de séjour (TSM) confirme les observations précédentes. Pour les deux derniers lots, le TSM intra-lot des particules de foin $(\mathrm{Yb})$ et de concentré $(\mathrm{Ce})$ n'a pas significativement différé, quel que soit le niveau alimentaire (tabl. 4).

TABLEAU 4

Temps de séjour moyen (TSM) chez la lapine adulte. Effet du régime et du niveau d'alimentation (Moyenne \pm écart type, exprimée en heures).

\begin{tabular}{|c|c|c|c|c|c|c|c|}
\hline & \multirow{2}{*}{ Lots } & \multicolumn{2}{|c|}{ Alimentation à volonté } & \multicolumn{2}{|c|}{ Alimentation rationnée } & \multicolumn{2}{|c|}{ Effet niveau alimentaire } \\
\hline & & $Y b(1)$ & $\mathrm{Ce}\left({ }^{2}\right)$ & $Y b$ (') & $\mathrm{Ce}(2)$ & $Y b$ & $\mathrm{Ce}$ \\
\hline Foin & $\begin{array}{l}\mathrm{x} \pm \sigma \\
\mathrm{n}\end{array}$ & $14,8 \pm 2,9$ & - & $17,0 \pm \frac{4,5}{4^{a}}$ & - & NS & - \\
\hline $\begin{array}{l}\text { Foin } \\
+ \\
\text { Pulpe }\end{array}$ & $\begin{array}{l}x \pm \sigma \\
n\end{array}$ & $16,6 \pm \frac{ \pm}{3} 2,8$ & $16,1 \pm 2,9$ & $20,1 \underset{5^{\mathrm{ab}}}{ \pm}$ & $19,1 \pm 2,0$ & $P<0,1$ & NS \\
\hline $\begin{array}{l}\text { Foin } \\
+ \\
\text { Son }\end{array}$ & $\begin{array}{l}\mathrm{x} \pm \sigma \\
\mathrm{n}\end{array}$ & $18,4 \underset{3}{ \pm} 2,0$ & $18,6 \pm \frac{2,2}{3}$ & $23,1 \underset{5^{b}}{ \pm} 2,9$ & $22,0 \pm \frac{2,0}{5}$ & * & $P<0,1$ \\
\hline \multicolumn{2}{|c|}{ Effet ration } & NS & NS & * & $P<0,1$ & & \\
\hline
\end{tabular}

(Résultats de l'analyse de variance à un facteur, " ration " ou " niveau alimentaire ").

$a, b$ : pour l'effet ration, les moyennes ayant une lettre commune ne diffèrent pas au seuil $P<0,05$ (Test de Newmann et Keuls).

$\mathrm{n}$ : effectifs contrôlés.

* : écart significatif au seuil $P<0,05$.

L'analyse de variance (ANVAR) à deux facteurs (ration et niveau alimentaire) montre un effet moyen de la ration sur le TSM très significatif $(P<0,01$ ). Pour analyser l'effet "ration " d'une même période, nous avons présenté au tableau 4 (et 3) les résultats de l'ANVAR à un facteur. On remarque ainsi que l'effet " ration » est plus marqué pendant P2 (écart significatif au seuil $5 \%$ : tableau 4). Un nombre de mesures plus élevé aurait sans doute permis d'observer des écarts significatifs (par l'ANVAR à un facteur) dans le cas de P1. Quand le son a remplacé une partie du foin, le TSM a augmenté d'environ $4 \mathrm{~h}$ à $6 \mathrm{~h}$ selon la période. Les TSM moyens des animaux ayant reçu la pulpe sont précisément à mi-chemin entre les TSM des deux autres lots $(16,3 \mathrm{~h}$ pendant $\mathrm{P} 1$ et $19,6 \mathrm{~h}$ en movenne durant $P 2$ ).

Le rationnement a entraîné un allongement du TSM de 2 à $4 \mathrm{~h}$ selon le lot. L'écart le plus important $(4 \mathrm{~h}$ ) est observé dans le cas du lot "Foin + Son ", soit un accroissement d'environ $26 \%$.

L'effet moyen de la ration, très significatif $(P<0,01)$ sur les valeurs de $E$ (tabl. 3), n'est pas significatif sur le temps d'excrétion de $5 \%$ du marqueur (t $5 \%$ ). Ce dernier ne diffère pas non plus selon le niveau d'alimentation. 


\section{Discussion.}

En alimentation ad libitum, les valeurs moyennes des TSM et des ingérés se classent de manière inverse entre les trois lots : TSM de $14,8-16,6$ et $18,4 \mathrm{~h}$ respectivement pour des ingérés secs de $169-134$ et $103 \mathrm{~g} \mathrm{MS} / \mathrm{j}$. Quand les animaux sont rationnés, le classement est encore le même. Mais les écarts entre lots du TSM sont plus élevés, alors que les écarts d'ingéré sont plus faibles. D'autre part, Fioramonti et al. (1978) montrent chez le lapin que la pulpe de betterave comparée à la luzerne déshydratée entraîne une inhibition de la motricité caecale et colique. Et cet effet est associé à une ingestion de pulpe plus faible que celle de la luzerne. Le niveau d'ingestion semble donc influencer le transit; mais ce n'est sans doute pas le seul facteur de variation du TSM.

En accord avec Fioramonti et al. (1978) et avec Pairet et al. (1986), nous observons que la pulpe de betterave et surtout le son de blé (mélangés à du foin) élèvent le temps de séjour des particules de foin (marquées à l'Yb). Ceci indique la présence d'une interaction entre le foin et le " concentré " quant au temps de séjour moyen de la ration dans le tube digestif. Une étude de digestibilité permettra de préciser l'effet de cette interaction sur l'utilisation digestive des rations. Signalons par ailleurs que cet effet de la ration ne s'explique pas par un début d'excrétion du marqueur plus tardif.

Ledin (1984) mesure, par la méthode des moments, le TSM à l'aide de chrome mordancé, chez des lapins en croissance recevant un aliment concentré : quant l'alimentation est restreinte à $60 \%$ de la consommation volontaire, le TSM passe de 12,6 à 19,7 $\mathrm{h}$. Dans notre cas, le rationnement élève le TSM de seulement $2 \mathrm{~h}$ à $4 \mathrm{~h}$ selon le lot. La richesse en fibres de nos rations peut stimuler la motricité digestive (Lebas et Laplace, 1977 ; Cherbut et al., 1984) et, par conséquent, atténuer en partie l'effet du rationnement.

Lebas et Laplace (1975) montrent qu'entre le $1^{\text {er }}$ et le $5^{\mathrm{e}}$ jour de rationnement, l'heure moyenne d'excrétion fécale est avancée d'environ $5 \mathrm{~h}$. Nos résultats indiquent en moyenne une avance d'environ 5 à $7 \mathrm{~h}$. La période de caecotrophie intervient donc plus tôt par rapport à l'heure d'administration du marqueur (to) et le TSM est alors plus élevé. L'allongement du temps de séjour par la caecotrophie avait déjà été montré par Piekarz (1963) et par Uden et al. (1982) sur des lapins porteurs de collier. L'effet du rationnement $s^{\prime}$ expliquerait-il surtout par une incidence plus forte de la caecotrophie ? Une réponse claire serait apportée par une mesure du TSM chez des animaux rationnés ou non et pour lesquels l'espace de temps entre to et la pratique de la caecotrophie serait identique.

Quoi qu'il en soit, notre technique de marquage a sans doute minimisé I'influence de la caecotrophie sur le TSM. En effet, nous avons fixé Yb et Ce sur la partie fibreuse des matières premières, relativement riche en particules grossières. Or, la caecotrophie est essentiellement un recyclage de fines particules (Bjöhrnag, 1972); elle influencerait donc assez peu le temps de séjour de nos marqueurs. Les travaux de Uden et Van Soest (1982) confirment cette hypothèse : ces auteurs montrent que, en l'absence de caecotrophie, le TSM des fines particules est plus faible, alors que celui des grosses particules reste inchangé par rapport à la période de contrôle. 
Pour des régimes similaires contenant les mêmes marqueurs, Poncet et al. (1986a) observent chez le mouton un allongement du temps de séjour de la ration $(F, F+S$, ou $F+P)$ lors du rationnement, en particulier dans le cas de la pulpe de betterave. Chez le lapin, c'est le son de blé qui présente la hausse de TSM la plus forte lors du rationnement $(+26 \%)$.

Chez le mouton et le taurillon, Poncet et al. (1987a) signalent pour des rations quasi-identiques un temps de séjour similaire entre le foin et le son des rations Foin et Foin + Son ; par contre, le transit de la pulpe est plus rapide que celui du foin.

Dans le cas de nos deux régimes «Foin-Concentré », les particules marquées par $\mathrm{Yb}$ ou Ce ont un TSM identique. Sur ce point, nous devons signaler que les deux fractions de la ration sont administrées sous forme broyée. Cela élimine donc des différences liées à la taille des particules (Laplace et Lebas, 1977). Pour cet essai, un éventuel temps de séjour différentiel des deux fractions n'aurait donc été justifié que par une différence de densité des particules ou de digestibilité. Toutefois, pour plus de précision, il serait nécessaire d'estimer dans quelles proportions $\mathrm{Yb}$ fixé sur le foin peut éventuellement se transférer sur le son au cours du transit (et inversement dans le cas du cérium), malgré les précautions prises lors de la fixation des marqueurs. En effet, les terres rares peuvent quitter leur site de fixation initial ainsi que Hartnell et Satter (1979), et Teeter et al. (1984) l'ont montré chez le ruminant. Chez le lapin, nous avons pu estimer que la proportion d'ytterbium susceptible de migrer serait de l'ordre de $10 \%$ (Gidenne, données non publiées).

Selon Poncet et al. (1987b), le temps de séjour d'une matière première dans le rumen ne serait pas uniquement déterminée par ses caractéristiques physiques et biochimiques, mais aussi par celle de l'ensemble de la ration; les différents composants de la ration pourraient former une masse fibreuse qui séquestrerait les particules plus fines (ou plus digestibles...) et freinerait leur évacuation. Dans le cas du lapin, nos résultats semblent indiquer un phénomène similaire : les deux types de constituants pariétaux (foin-concentré) formeraient un ensemble homogène déterminant le temps de séjour de la ration dans son ensemble.

En conclusion, l'addition de pulpe de betterave et plus encore de son de blé, à une ration constituée de foin $(90 \%)$, entraîne une élévation du TSM du foin et de la ration dans son ensemble. Nous ne constatons pas d'écart du temps de séjour des principaux constituants fibreux d'une même ration en fonction de leur origine botanique. Le rationnement entraîne un décalage des périodes d'excrétion fécale et la caecotrophie intervient plus précocément au cours de l'élimination fécale des marqueurs. Les valeurs du TSM s'accroissent alors, d'environ 15 à $20 \%$.

Recu en novembre 1986. Accepté en mars 1987.

Remerciements. - Cette étude a été réalisée dans le cadre d'une Action Thématique Programmée (I.N.R.A.), dont le thème était l'utilisation digestive des constituants pariétaux chez différentes espèces d'herbivores. 
Résumé.

L'influence de l'addition d'une matière première concentrée riche en fibres (son de blé ou pulpe de betterave) à un aliment à base de foin (mélange dactyle-luzerne : 50/50), sur le transit de ces deux fractions (foin et concentré) est mesurée sur trois lots de 8 lapines adultes (Néozélandais Blanc $\times$ Californien). Les trois régimes composés à $90 \%$ de foin seul, de foin + son, ou de foin + pulpe, sont distribués à deux niveaux alimentaires : ad libitum et aux $2 / 3$ de la consommation ad libitum. Le temps de séjour des deux fractions principales est mesuré à l'aide de deux marqueurs : I'ytterbium-169 fixé sur le foin, et le cérium-141 fixé sur le son ou la pulpe.

Les animaux recevant le régime «foin " seul excrètent plus de $90 \%$ du marqueur pendant les premières 24 heures. Le temps de séjour moyen (TSM) de cet aliment est le plus bref : 14,8 h en moyenne. L'addition de pulpe ou de son augmente le TSM de la ration, de 2 et $4 \mathrm{~h}$, respectivement. Le rationnement avance de 5 à $7 \mathrm{~h}$ la période de pratique de la caecotrophie. Ceci entraîne un allongement du TSM de 2 à $4 \mathrm{~h}$ selon le lot.

Dans le cas des deux rations Foin + "Concentré ", les courbes d'excrétion de ${ }^{169} \mathrm{Yb}$ et du ${ }^{141} \mathrm{Ce}$ sont quasi identiques. Les particules de foin et de "concentré " auraient donc un temps de séjour moyen similaire (écarts non significatifs).

\section{Références}

BJÖRNHAG G., 1972. Separation and delay of contents in the rabbit colon. Swedish. J. agric. Res., 2, 125.

BLAXTER K. L., GRAHAM N. Mc C., WAINMAN F. W., 1956. Some observations on the digestibility of food by sheep and on related problems. Br. J. Nutr., 10, 69-91.

CHERBUT C., BARRY J. L., GUENEAU S., 1984. Mode d'action des fibres alimentaires sur le transit digestif : hypothèses physiques. Repr. Nutr. Dévelop., 24, 771.

COLIN M., ARKHURST G., LEBAS F., 1973. Effets de I'addition de méthionine au régime alimentaire sur les performances de croissance chez le lapin. Ann. Zootech., 22, 485-491.

ELLIS W. C., BEEVER D. E., 1985. Methods for binding rare-earths to specific feed particles, 154165. In P. M. KENNEDY. Techniques in particle size analysis of feed and digesta in ruminants. Can. Soc. anim. Sci. Edmonton.

FIORAMONTI J., BUÉNO L., CANDAU M., 1978. Motricité digestive et glucides membranaires

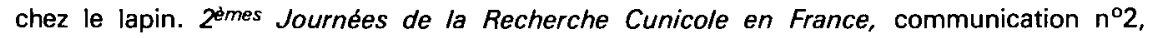
4-5 avril, Toulouse, France.

HARTNELL G. F., SATTER L. D., 1979. Extent of particulate marker (samarium, lanthanum, cerium) movement from one digesta particle to another. J. anim. Sci. 48, 375.

LAPLACE J. P., LEBAS F., RIOPEREZ J., 1974. Le transit digestif chez le lapin. I. Utilisation du cérium 141 : étude méthodologique et descriptive. Ann. Zootech., 23, 555-576.

LAPLACE J. P., LEBAS F., 1975. Le transit digestif chez le lapin. III. Influence de l'heure et du mode d'administration sur l'excrétion fécale du cérium 141, chez le lapin alimenté ad libitum. Ann. Zootech., 24, 255-265.

LAPLACE J. P., LEBAS F., 1977. Le transit digestif chez le Lapin. VII. Influence de la finesse de broyage des constituants d'un aliment granulé. Ann. Zootech., 26, 413-420.

LEBAS F., LAPLACE J. P., 1975. Le transit digestif chez le lapin. V. Evolution de l'excrétion fécale en fonction de l'heure de distribution de l'aliment et du niveau de rationnement durant les 5 jours qui suivent l'application de ce dernier. Ann. Zootech., 24, 613-627.

LEBAS F., LAPLACE J. P., 1977. Le transit digestif chez le lapin. VIII. Influence de la source de cellulose. Ann. Zootech., 26, 575-584.

LEDIN I., 1984. A note on the effect of different feeding levels on the rate of digesta passage in rabbits. Acta agric. scand., 34, 67-70.

PAIRET M., BOUYSSOU Th., AUVERGNE A., CANDAU M., RUCKEBUSCH Y., 1986. Stimulation physico-chimique d'origine alimentaire et motricité digestive chez le lapin. Repr. Nutr. Dévelop., 26, 85-95. 
PIEKARZ R., 1963. (Effet de la coprophagie sur le temps de transit digestif chez le lapin domestique.) Acta. physiol. polon., 14, 359-370.

PONCET C., GOMEZ L., MICHALET-DOREAU B., GEAY Y., 1987a. Temps de séjour comparé du foin, du son de blé et de la pulpe de betterave, d'une ration mixte distribuée à deux niveaux d'alimentation à des moutons et des taurillons. z'mes Journées Rech. Nutr. Alim. des Herbivores, 19-20 mars 1986, communication $n^{\circ} 3$. Repr. Nutr. Dévelop., 27, 219-220.

PONCET C., GONZALES J., MICHALET-DOREAU B., 1987b. Effet du pourcentage de concentré de la ration et du niveau d'ingestion sur la vitesse de passage dans le rumen de différents types d'aliments chez le mouton. 2èmes Journées Rech. Nutr. Alim. des Herbivores, 19-20 mars 1986, Paris, communication $n^{\circ}$ 21. Repr. Nutr. Dévelop., 27, 257-258.

TEETER R. G., OWENS F. N., MADER T. L., 1984. Ytterbium chloride as a marker for particulate marker in the rumen. J. anim. Sci., 58, 465-473.

THIELMANS M.-F., FRANC̣OIS E., BODART C., THÉWIS A., 1978. Mesures du transit gastrointestinal chez le porc à l'aide de radiolanthanides. Comparaison avec le mouton. Ann. Biol. anim. Bioch. Biophys., 18, 237-247.

UDEN P., ROUNSAVILLE T. R., WIGGANS G. R., VAN SOEST P. J., 1982 . The measurement of liquid and solid digesta retention in ruminants, equines and rabbits given timothy (Phleum partensel hay. Br. J. Nutr., 48, 329-339.

UDEN P., VAN SOEST P. J., 1982. The determination of digesta particle size in some herbivores. Anim. Feed. Sci. Technol., 7, 35-44. 